\title{
On Geometric Constants for Discrete Morrey Spaces
}

\author{
Adam Adam, Hendra Gunawan*৫e \\ Analysis and Geometry Group, Faculty of Mathematics and Natural Sciences, \\ Bandung Institute of Technology, Bandung 40132, Indonesia \\ adam_adam@students.itb.ac.id, hgunawan@math.itb.ac.id \\ *Correspondence: hgunawan@math.itb.ac.id
}

\begin{abstract}
AвStRACT. In this paper we prove that the $n$-th Von Neumann-Jordan constant and the $n$-th James constant for discrete Morrey spaces $\ell_{q}^{p}$ where $1 \leq p<q<\infty$ are both equal to $n$. This result tells us that the discrete Morrey spaces are not uniformly non- $\ell^{1}$, and hence they are not uniformly $n$-convex.
\end{abstract}

\section{INTRODUCTION}

Let $n \geq 2$ be a non-negative integer and $(X,\|\cdot\|)$ be a Banach space. The $n$-th Von NeumannJordan constant for $X[6]$ is defined by

$$
C_{N J}^{(n)}(X):=\sup \left\{\frac{\sum_{ \pm}\left\|u_{1} \pm u_{2} \pm \cdots \pm u_{n}\right\|_{X}^{2}}{2^{n-1} \sum_{i=1}^{n}\left\|u_{i}\right\|_{X}}: u_{i} \neq 0, i=1,2, \ldots, n\right\}
$$

and the $n$-th James constant for $X[7]$ is defined by

$$
C_{J}^{(n)}(X):=\sup \left\{\min \left\|u_{1} \pm u_{2} \pm \cdots \pm u_{n}\right\|: u_{i} \in S_{X}, i=1,2, \ldots, n\right\} .
$$

Note that in the definition of $C_{N J}^{(n)}(X)$, the sum $\Sigma_{ \pm}$is taken over all possible combinations of \pm signs. Similarly, in the definition of $C_{J}^{(n)}(X)$, the minimum is taken over all possible combinations of \pm signs, while the supremum is taken over all $u_{i}^{\prime} \mathrm{s}$ in the unit sphere $S_{X}:=\{u \in X:\|u\|=1\}$. These constants measure some sort of convexity of a Banach space.

We say that $X$ is uniformly $n$-convex [2] if for every $\varepsilon \in(0, n]$ there exists a $\delta \in(0,1)$ such that for every $u_{1}, u_{2}, \ldots, u_{n} \in S_{X}$ with $\left\|u_{1} \pm u_{2} \pm \cdots \pm u_{n}\right\| \geq \varepsilon$ for all combinations of \pm signs except for $\left\|u_{1}+u_{2}+\cdots+u_{n}\right\|$, we have

$$
\left\|u_{1}+u_{2}+\cdots+u_{n}\right\| \leq n(1-\delta) .
$$

Received: 31 Aug 2021.

Key words and phrases. $n$-th Von Neumann-Jordan constant; $n$-th James constant; discrete Morrey spaces; uniformly non- $\ell^{1}$ spaces; uniformly $n$-convex spaces. 
Meanwhile, we say that $X$ is uniformly non- $\ell_{n}^{1}[1,5,8]$ if there exists a $\delta \in(0,1)$ such that for every $u_{1}, u_{2}, \ldots, u_{n} \in S_{X}$ we have

$$
\min \left\|u_{1} \pm u_{2} \pm \cdots \pm u_{n}\right\| \leq n(1-\delta)
$$

Note that for $n=2$, uniformly non- $\ell_{n}^{1}$ spaces are known as uniformly nonsquare spaces, while for $n=3$ they are known as uniformly non-octahedral spaces. One may verify that if $X$ is uniformly $n$-convex, then $X$ is uniformly non- $\ell_{n}^{1}[2]$.

Now a few remarks about the two constants, and their associations with the uniformly non- $\ell_{n}^{1}$ and uniformly $n$-convex properties.

- $1 \leq C_{N J}^{(n)}(X) \leq n$ and $C_{N J}^{(n)}(X)=1$ if and only if $X$ is a Hilbert space [6].

- $1 \leq C_{J}^{(n)}(X) \leq n$. If $\operatorname{dim}(X)=\infty$, then $\sqrt{n} \leq C_{J}^{(n)}(X) \leq n$. Moreover, if $X$ is a Hilbert space, then $C_{J}^{(n)}(X)=\sqrt{n}[7]$.

- $X$ is uniformly non- $\ell_{n}^{1}$ if and only if $C_{N J}^{(n)}(X)<n[6]$.

- $X$ is uniformly non- $\ell_{n}^{1}$ if and only if $C_{j}^{(n)}(X)<n[7]$.

The last two statements tell us that if $C_{N J}^{(n)}(X)=n$ or $C_{J}^{(n)}(X)=n$, then $X$ is not uniformly non- $\ell_{n}^{1}$ and hence not uniformly $n$-convex.

In this paper, we shall compute the value of the two constants for discrete Morrey spaces. Let $\omega:=\mathbb{N} \cup\{0\}$ and $m=\left(m_{1}, m_{2}, \ldots, m_{d}\right) \in \mathbb{Z}^{d}$. Define

$$
S_{m, N}:=\left\{k \in \mathbb{Z}^{d}:\|k-m\|_{\infty} \leq N\right\}
$$

where $N \in \omega$ and $\|m\|_{\infty}=\max \left\{\left|m_{i}\right|: 1 \leq i \leq d\right\}$. Denote by $\left|S_{m, N}\right|$ the cardinality of $S_{m, N}$ for $m \in \mathbb{Z}^{d}$ and $N \in \omega$. Then we have $\left|S_{m, N}\right|=(2 N+1)^{d}$.

Now let $1 \leq p \leq q<\infty$. Define $\ell_{q}^{p}=\ell_{q}^{p}\left(\mathbb{Z}^{d}\right)$ to be the discrete Morrey space as introduced in [3], which consists of all sequences $x: \mathbb{Z}^{d} \rightarrow \mathbb{R}$ with

$$
\|x\|_{\ell_{q}^{p}}:=\sup _{m \in \mathbb{Z}^{d}, N \in \omega}\left|S_{m, N}\right|^{\frac{1}{q}-\frac{1}{p}}\left(\sum_{k \in S_{m, N}}\left|x_{k}\right|^{p}\right)^{\frac{1}{p}}<\infty,
$$

where $x:=\left(x_{k}\right)$ with $k \in \mathbb{Z}^{d}$. One may observe that these discrete Morrey spaces are Banach spaces [3]. Note, in particular, that for $p=q$, we have $\ell_{q}^{p}=\ell^{q}$.

From [4] we already know that $C_{N J}\left(\ell_{q}^{p}\right)=C_{J}\left(\ell_{q}^{p}\right)=2$ for $1 \leq p<q<\infty$, which implies that $\ell_{q}^{p}$ are not uniformly nonsquares for those $p^{\prime} s$ and $q$ 's. In this paper, we shall show that $C_{N J}^{(n)}\left(\ell_{q}^{p}\right)=C_{J}^{(n)}\left(\ell_{q}^{p}\right)=n$ for $1 \leq p<q<\infty$, which leads us to the conclusion that $\ell_{q}^{p}$ are not uniformly non- $\ell_{n}^{1}$ for those $p^{\prime}$ s and $q$ 's, which is sharper than the existing result. (If $X$ is not uniformly non- $\ell_{n}^{1}$, then $X$ is not uniformly non $\ell_{n-1}^{1}$, provided that $n \geq 3$.) 


\section{Main Results}

The value of the $n$-th Von Neumann-Jordan constant and the $n$-th James constant for discrete Morrey spaces are stated in the following theorems. To understand the idea of the proof, we first present the result for $n=3$.

Theorem 2.1. For $1 \leq p<q<\infty$, we have $C_{N J}^{(3)}\left(\ell_{q}^{p}\left(\mathbb{Z}^{d}\right)\right)=C_{J}^{(3)}\left(\ell_{q}^{p}\left(\mathbb{Z}^{d}\right)\right)=3$.

Proof. To prove the theorem, it suffices for us to find $x^{(1)}, x^{(2)}, x^{(3)} \in \ell_{q}^{p}$ such that

$$
\frac{\sum_{ \pm}\left\|x^{(1)} \pm x^{(2)} \pm x^{(3)}\right\|_{\ell_{q}^{p}}^{2}}{2^{2} \sum_{i=1}^{3}\left\|x^{(i)}\right\|_{\ell_{q}^{p}}}=3
$$

for the Von Neumann-Jordan constant, and

$$
\min \left\|x^{(1)} \pm x^{(2)} \pm x^{(3)}\right\|_{\ell_{q}^{p}}=3
$$

for the James constant.

Case 1: $d=1$. Let $j \in \mathbb{Z}$ be a nonnegative, even integer such that $j>4^{\frac{q}{q-p}}-1$, or equivalently

$$
(j+1)^{\frac{1}{q}-\frac{1}{p}}<4^{-\frac{1}{p}} .
$$

Construct $x^{(1)}, x^{(2)}, x^{(3)} \in \ell_{q}^{p}(\mathbb{Z})$ as follows:

- $x^{(1)}=\left(x_{k}^{(1)}\right)_{k \in \mathbb{Z}}$ is defined by

$$
x_{k}^{(1)}= \begin{cases}1, & k=0, j, 2 j, 3 j \\ 0, & \text { otherwise }\end{cases}
$$

- $x^{(2)}=\left(x_{k}^{(2)}\right)_{k \in \mathbb{Z}}$ is defined by

$$
x_{k}^{(2)}= \begin{cases}1, & k=0, j \\ -1, & k=2 j, 3 j \\ 0, & \text { otherwise }\end{cases}
$$

- $x^{(3)}=\left(x_{k}^{(3)}\right)_{k \in \mathbb{Z}}$ is defined by

$$
x_{k}^{(3)}= \begin{cases}1, & k=0,2 j \\ -1, & k=j, 3 j \\ 0, & \text { otherwise }\end{cases}
$$


The three sequences are in the unit sphere of $\ell_{q}^{p}(\mathbb{Z})$. Indeed, for the first sequence, we have

$$
\begin{aligned}
\left\|x^{(1)}\right\|_{\ell_{q}^{p}} & =\sup _{m \in \mathbb{Z}, N \in \omega}\left|S_{m, N}\right|^{\frac{1}{q}-\frac{1}{p}}\left(\left.\sum_{k \in S_{m, N}}\left|x_{k}^{(1)}\right|^{p}\right|^{\frac{1}{p}}\right. \\
& =\sup _{m \in \mathbb{Z} \cap[0,3 j], N \in \mathbb{Z} \cap[0,3 j / 2]}\left|S_{m, N}\right|^{\frac{1}{q}-\frac{1}{p}}\left(\sum_{k \in S_{m, N}}\left|x_{k}^{(1)}\right|^{p}\right)^{\frac{1}{p}} \\
& =\max \left\{1,(j+1)^{\frac{1}{q}-\frac{1}{p}} 2^{\frac{1}{p}},(2 j+1)^{\frac{1}{q}-\frac{1}{p}} 3^{\frac{1}{p}},(3 j+1)^{\frac{1}{q}-\frac{1}{p}} 4^{\frac{1}{p}}\right\} .
\end{aligned}
$$

Since $(3 j+1)^{\frac{1}{q}-\frac{1}{p}}<(2 j+1)^{\frac{1}{q}-\frac{1}{p}}<(j+1)^{\frac{1}{q}-\frac{1}{p}}<4^{-\frac{1}{p}}$, we get $\left\|x^{(1)}\right\|_{\ell_{q}^{p}}=1$. Similarly, one may observe that $\left\|x^{(2)}\right\|_{\ell_{q}^{p}}=\left\|x^{(3)}\right\|_{\ell_{q}^{p}}=1$.

Next, we observe that

$$
\begin{aligned}
& x_{k}^{(1)}+x_{k}^{(2)}+x_{k}^{(3)}= \begin{cases}3, & k=0, \\
1, & k=j, 2 j, \\
-1, & k=3 j, \\
0, & \text { otherwise; }\end{cases} \\
& x_{k}^{(1)}+x_{k}^{(2)}-x_{k}^{(3)}= \begin{cases}3, & k=j, \\
1, & k=0,3 j, \\
-1, & k=2 j, \\
0, & \text { otherwise; }\end{cases} \\
& x_{k}^{(1)}-x_{k}^{(2)}+x_{k}^{(3)}= \begin{cases}3, & k=2 j, \\
1, & k=0,3 j, \\
-1, & k=j, \\
0, & \text { otherwise; }\end{cases} \\
& x_{k}^{(1)}-x_{k}^{(2)}-x_{k}^{(3)}= \begin{cases}3, & k=3 j, \\
1, & k=j, 2 j, \\
-1, & k=0, \\
0, & \text { otherwise. }\end{cases}
\end{aligned}
$$

We first compute that

$$
\left\|x^{(1)}+x^{(2)}+x^{(3)}\right\|_{\ell_{q}^{p}}=\max \left\{3,(j+1)^{\frac{1}{q}-\frac{1}{p}}\left(3^{p}+1\right)^{\frac{1}{p}},(2 j+1)^{\frac{1}{q}-\frac{1}{p}}\left(3^{p}+2\right)^{\frac{1}{p}},(3 j+1)^{\frac{1}{q}-\frac{1}{p}}\left(3^{p}+3\right)^{\frac{1}{p}}\right\} .
$$

Notice that

$$
\begin{aligned}
& \text { - }(j+1)^{\frac{1}{a}-\frac{1}{p}}\left(3^{p}+1\right)^{\frac{1}{p}}<\left(\frac{3^{p}+1^{p}}{4}\right)^{\frac{1}{p}}<\left(3^{p}\right)^{\frac{1}{p}}=3 . \\
& \text { - }(2 j+1)^{\frac{1}{q}-\frac{1}{p}}\left(3^{p}+2\right)^{\frac{1}{p}}<(j+1)^{\frac{1}{q}-\frac{1}{p}}\left(3^{p}+2\right)^{\frac{1}{p}}<\left(\frac{3^{p}+2}{4}\right)^{\frac{1}{p}}<3 .
\end{aligned}
$$


- $(3 j+1)^{\frac{1}{q}-\frac{1}{p}}\left(3^{p}+3\right)^{\frac{1}{p}}<(j+1)^{\frac{1}{q}-\frac{1}{p}}\left(3^{p}+3\right)^{\frac{1}{p}}<\left(\frac{3^{p}+3}{4}\right)^{\frac{1}{p}}<3$.

Hence, we obtain $\left\|x^{(1)}+x^{(2)}+x^{(3)}\right\|_{\ell_{q}^{p}}=3$.

Similarly, we have

$$
\left\|x^{(1)} \pm x^{(2)} \pm x^{(3)}\right\|_{\ell_{q}^{p}}=\sup _{m \in \mathbb{Z} \cap[0,3 j], N \in \mathbb{Z} \cap[0,3 j / 2]}\left|S_{m, N}\right|^{\frac{1}{q}-\frac{1}{p}}\left(\sum_{k \in S_{m, N}}\left|x_{k}^{(1)} \pm x_{k}^{(2)} \pm x_{k}^{(3)}\right|^{p}\right)^{\frac{1}{p}}=3
$$

for every combination of \pm signs.

Consequently, $\frac{\sum_{ \pm}\left\|x^{(1)} \pm x^{(2)} \pm x^{(3)}\right\|_{\ell_{q}^{p}}^{2}}{2^{2} \sum_{i=1}^{3}\left\|x^{(i)}\right\|_{\ell_{q}^{p}}}=3$ and $\min \left\|x^{(1)} \pm x^{(2)} \pm x^{(3)}\right\|_{\ell_{q}^{p}}=3$, so we come to the conclusion that

$$
C_{N J}^{(3)}\left(\ell_{q}^{p}(\mathbb{Z})\right)=C_{J}^{(3)}\left(\ell_{q}^{p}(\mathbb{Z})\right)=3
$$

Case 2: $d>1$. Let $j \in \mathbb{Z}$ be a nonnegative, even integer such that $j>4^{\frac{q}{d(q-p)}}-1$, which is equivalent to

$$
(j+1)^{d\left(\frac{1}{q}-\frac{1}{p}\right)}<4^{-\frac{1}{p}} .
$$

We then construct $x^{(1)}, x^{(2)}, x^{(3)} \in \ell_{q}^{p}\left(\mathbb{Z}^{d}\right)$ as follows:

- $x^{(1)}=\left(x_{k}^{(1)}\right)_{k \in \mathbb{Z}^{d}}$ is defined by

$$
x_{k}^{(1)}= \begin{cases}1, & k=(0,0, \ldots, 0),(j, 0, \ldots, 0),(2 j, 0, \ldots, 0),(3 j, 0, \ldots, 0), \\ 0, & \text { otherwise }\end{cases}
$$

- $x^{(2)}=\left(x_{k}^{(2)}\right)_{k \in \mathbb{Z}^{d}}$ is defined by

$$
x_{k}^{(2)}= \begin{cases}1, & k=(0,0, \ldots, 0),(j, 0, \ldots, 0), \\ -1, & k=(2 j, 0, \ldots, 0),(3 j, 0, \ldots, 0), \\ 0, & \text { otherwise }\end{cases}
$$

- $x^{(3)}=\left(x_{k}^{(3)}\right)_{k \in \mathbb{Z}^{d}}$ is defined by

$$
x_{k}^{(3)}= \begin{cases}1, & k=(0,0, \ldots, 0),(2 j, 0, \ldots, 0), \\ -1, & k=(j, 0, \ldots, 0),(3 j, 0, \ldots, 0), \\ 0, & \text { otherwise }\end{cases}
$$

As in the case where $d=1$, one may observe that

$$
\begin{aligned}
\left\|x^{(1)}\right\|_{\ell_{q}^{p}} & =\sup _{m \in \mathbb{Z}^{d}, N \in \omega}\left|S_{m, N}\right|^{\frac{1}{q}-\frac{1}{p}}\left(\sum_{k \in S_{m, N}}\left|x_{k}^{(1)}\right|^{p}\right)^{\frac{1}{p}} \\
& =\max \left\{1,(j+1)^{d\left(\frac{1}{q}-\frac{1}{p}\right)} 2^{\frac{1}{p}},(2 j+1)^{d\left(\frac{1}{q}-\frac{1}{p}\right)} 3^{\frac{1}{p}},(3 j+1)^{d\left(\frac{1}{q}-\frac{1}{p}\right)} 4^{\frac{1}{p}}\right\} \\
& =1 .
\end{aligned}
$$


We also get $\left\|x^{(2)}\right\|_{\ell_{q}^{p}}=\left\|x^{(3)}\right\|_{\ell_{q}^{p}}=1$. Moreover, through similar observation as in the 1 -dimensional case, we have

$$
\left\|x^{(1)} \pm x^{(2)} \pm x^{(3)}\right\|_{\ell_{q}^{p}}=3
$$

for every possible combinations of \pm signs. It thus follows that

$$
C_{J}^{(3)}\left(\ell_{q}^{p}\left(\mathbb{Z}^{d}\right)\right)=\sup \left\{\min \left\|x_{1} \pm x_{2} \pm x_{3}\right\|_{\ell_{q}^{p}}: x_{1}, x_{2}, x_{3} \in S_{\ell_{q}^{p}}\right\}=3
$$

and

$$
C_{N J}^{(3)}\left(\ell_{q}^{p}\left(\mathbb{Z}^{d}\right)\right)=\sup \left\{\frac{\sum_{ \pm}\left\|x_{1} \pm x_{2} \pm x_{3}\right\|_{\ell_{q}^{p}}^{2}}{2^{2} \sum_{i=1}^{3}\left\|x_{i}\right\|_{\ell_{q}^{p}}}: x_{i} \neq 0, i=1,2,3\right\}=3 .
$$

We now state the general result for $n \geq 3$. (The proof is also valid for $n=2$, which amounts to the work of [3].)

Theorem 2.2. For $1 \leq p<q<\infty$, we have $C_{N J}^{(n)}\left(\ell_{q}^{p}\left(\mathbb{Z}^{d}\right)\right)=C_{J}^{(n)}\left(\ell_{q}^{p}\left(\mathbb{Z}^{d}\right)\right)=n$.

Proof. As for $n=3$, we shall consider the case where $d=1$ first, and then the case where $d>1$ later.

Case 1: $d=1$. Let $j \in \mathbb{Z}$ be a nonnegative, even integer such that $j>2^{(n-1)\left(\frac{q}{q-p}\right)}-1$, which is equivalent to

$$
(j+1)^{\frac{1}{q}-\frac{1}{p}}<2^{-\frac{(n-1)}{p}} .
$$

We construct $x^{(i)} \in \ell_{q}^{p} \in \mathbb{Z}$ for $i=1,2, \ldots, n$ as follows:

- $x^{(1)}=\left(x_{k}^{(1)}\right)_{k \in \mathbb{Z}}$ is defined by

$$
x_{k}^{(1)}= \begin{cases}1, & k \in S_{1}^{(1)} \\ 0, & \text { otherwise }\end{cases}
$$

where

$$
S_{1}^{(1)}=\left\{0, j, 2 j, 3 j, \ldots,\left(2^{n-1}-1\right) j\right\} ;
$$

- $x^{(i)}=\left(x_{k}^{(i)}\right)_{k \in \mathbb{Z}}$ for $2 \leq i \leq n$ is defined by

$$
x_{k}^{(i)}= \begin{cases}1, & k \in S_{1}^{(i)} \\ -1, & k \in S_{-1}^{(i)} \\ 0, & \text { otherwise }\end{cases}
$$

with the following rules: Write $P=\left\{0, j, 2 j, \ldots,\left(2^{n-1}-1\right) j\right\}$ as

$$
P=P_{1}^{(i)} \cup P_{2}^{(i)} \cup \cdots \cup P_{2^{i-1}}^{(i)}
$$


where $P_{1}^{(i)}$ consists of the first $\frac{2^{n-1}}{2^{i-1}}$ terms of $P, P_{2}^{(i)}$ consists of the next $\frac{2^{n-1}}{2^{i-1}}$ terms of $P$, and so on. Then $S_{1}^{(i)}$ and $S_{-1}^{(i)}$ are given by

$$
\begin{aligned}
& S_{1}^{(i)}=P_{1}^{(i)} \cup P_{3}^{(i)} \cup \cdots \cup P_{2^{i-1}-1}^{(i)}, \\
& S_{-1}^{(i)}=P_{2}^{(i)} \cup P_{4}^{(i)} \cup \cdots \cup P_{2^{i-1}}^{(i)} .
\end{aligned}
$$

For example, for $i=2, x^{(2)}=\left(x_{k}^{(2)}\right)_{k \in \mathbb{Z}}$ is defined by

$$
x_{k}^{(2)}= \begin{cases}1, & k \in S_{1}^{(2)} \\ -1, & k \in S_{-1}^{(2)} \\ 0, & \text { otherwise }\end{cases}
$$

where

$$
\begin{aligned}
& S_{1}^{(2)}=\left\{0, j, 2 j, 3 j, \ldots,\left(\frac{2^{n-1}}{2}-1\right) j\right\} \\
& S_{-1}^{(2)}=\left\{\left(\frac{2^{n-1}}{2}\right) j,\left(\frac{2^{n-1}}{2}+1\right) j, \ldots,\left(2^{n-1}-1\right) j\right\}
\end{aligned}
$$

Note that the largest absolute value of the terms of $x^{(i)}$ in the above construction will be equal to 1 for each $i=1, \ldots, n$. Next, since the number of possible combinations of \pm signs in $x^{(1)} \pm x^{(2)} \pm \cdots \pm x^{(n)}$ is $2^{n-1}$, the above construction will give us $1+1+\cdots+1=n$ as the largest absolute value of $x^{(1)} \pm x^{(2)} \pm \cdots \pm x^{(n)}$ for every combination of \pm signs. This means that, if $x^{(1)} \pm x^{(2)} \pm \cdots \pm x^{(n)}=\left(x_{k}\right)_{k \in \mathbb{Z}}$, then $\max _{k \in \mathbb{Z}}\left|x_{k}\right|=n$.

Let us now compute the norms. For $x^{(1)}$, we have

$$
\begin{aligned}
\left\|x^{(1)}\right\|_{\ell_{q}^{p}} & =\sup _{m \in \mathbb{Z}, N \in \omega}\left|S_{m, N}\right|^{\frac{1}{q}-\frac{1}{p}}\left(\sum_{k \in S_{m, N}}\left|x_{k}^{(1)}\right|^{p}\right)^{\frac{1}{p}} \\
& =\sup _{m \in \mathbb{Z} \cap\left[0,\left(2^{n-1}-1\right) j\right], N \in \mathbb{Z} \cap\left[0,\left(2^{n-1}-1\right) j / 2\right]}\left|S_{m, N}\right|^{\frac{1}{q}-\frac{1}{p}}\left(\sum_{k \in S_{m, N}}\left|x_{k}^{(1)}\right|^{p}\right)^{\frac{1}{p}} \\
& =\max \left\{1,(j+1)^{\frac{1}{q}-\frac{1}{p}} 2^{\frac{1}{p}},(2 j+1)^{\frac{1}{q}-\frac{1}{p}} 3^{\frac{1}{p}}, \ldots,\left(\left(2^{n-1}-1\right) j+1\right)^{\frac{1}{q}-\frac{1}{p}} 2^{\frac{n-1}{p}}\right\} .
\end{aligned}
$$

For each $r=1,2, \ldots, 2^{n-1}-1$, we have $(r j+1)^{\frac{1}{q}-\frac{1}{p}} \leq(j+1)^{\frac{1}{q}-\frac{1}{p}}$ and $(r+1)^{\frac{1}{p}} \leq 2^{\frac{n-1}{p}}$, so that

$$
(r j+1)^{\frac{1}{q}-\frac{1}{p}}(r+1)^{\frac{1}{p}} \leq(j+1)^{\frac{1}{q}-\frac{1}{p}} 2^{\frac{n-1}{p}}<2^{-\frac{n-1}{p}} 2^{\frac{n-1}{p}}=1 .
$$

Hence we obtain $\left\|x^{(1)}\right\|_{\ell_{q}^{p}}=1$. Similarly, one may verify that

$$
\left\|x^{(2)}\right\|_{\ell_{q}^{p}}=\left\|x^{(3)}\right\|_{\ell_{q}^{p}}=\cdots=\left\|x^{(n)}\right\|_{\ell_{q}^{p}}=1
$$


Next, we shall compute the norms of $x^{(1)} \pm x^{(2)} \pm \cdots \pm x^{(n)}$. Write $x^{(1)}+x^{(2)}+\cdots+x^{(n)}=\left(x_{k}\right)_{k \in \mathbb{Z}}$ where

$$
x_{k}:= \begin{cases}a_{1}, & k=0, \\ a_{2}, & k=j, \\ a_{3}, & k=2 j, \\ \vdots & \\ a_{2^{n-1},} & k=\left(2^{n-1}-1\right) j, \\ 0, & \text { otherwise, }\end{cases}
$$

with $a_{1}=n$ and $\left|a_{i}\right|<n$ for $i=2,3, \ldots,\left(2^{n-1}\right) j$. Accordingly, we have

$$
\begin{aligned}
\left\|x^{(1)}+x^{(2)}+\cdots+x^{(n)}\right\|_{\ell_{q}^{p}}= & \sup _{m \in \mathbb{Z}, N \in \omega}\left|S_{m, N}\right|^{\frac{1}{q}-\frac{1}{p}}\left(\sum_{k \in S_{m, N}}\left|x_{k}\right|^{p}\right)^{\frac{1}{p}} \\
= & \sup _{m \in \mathbb{Z} \cap\left[0,\left(2^{n-1}-1\right) j\right], N \in \mathbb{Z} \cap\left[0,\left(2^{n-1}-1\right) j / 2\right]}\left|S_{m, N}\right|^{\frac{1}{q}-\frac{1}{p}}\left(\sum_{k \in S_{m, N}}\left|x_{k}\right|^{p}\right)^{\frac{1}{p}} \\
= & \max \left\{n,(j+1)^{\frac{1}{q}-\frac{1}{p}}\left(n^{p}+a_{2}^{p}\right)^{\frac{1}{p}},(2 j+1)^{\frac{1}{q}-\frac{1}{p}}\left(n^{p}+a_{2}^{p}+a_{3}^{p}\right)^{\frac{1}{p}}\right. \\
& \left.\ldots,\left(\left(2^{n-1}-1\right) j+1\right)^{\frac{1}{q}-\frac{1}{p}}\left(n^{p}+\sum_{i=2}^{2^{n-1}} a_{i}^{p}\right)^{\frac{1}{p}}\right\} .
\end{aligned}
$$

Since $(r j+1)^{\frac{1}{q}-\frac{1}{p}} \leq(j+1)^{\frac{1}{q}-\frac{1}{p}}$ for each $r=1,2, \ldots, 2^{n-1}-1$, we obtain

$$
\begin{aligned}
(r j+1)^{\frac{1}{q}-\frac{1}{p}}\left(n^{p}+\sum_{i=2}^{r+1} a_{i}^{p}\right)^{\frac{1}{p}} & \leq(j+1)^{\frac{1}{q}-\frac{1}{p}}\left(n^{p}+\sum_{i=2}^{r+1} a_{i}^{p}\right)^{\frac{1}{p}} \\
& <2^{-\frac{(n-1)}{p}}\left(n^{p}+\sum_{i=2}^{r+1} a_{i}^{p}\right)^{\frac{1}{p}} \\
& <2^{-\frac{(n-1)}{p}}(\underbrace{n^{p}+n^{p}+\cdots+n^{p}}_{r+1 \text { times }})^{\frac{1}{p}} \\
& =2^{-\frac{(n-1)}{p}}(r+1)^{\frac{1}{p}}\left(n^{p}\right)^{\frac{1}{p}} \\
& \leq 2^{-\frac{(n-1)}{p}} 2^{\frac{(n-1)}{p}} n \\
& =n .
\end{aligned}
$$

It thus follows that

$$
\left\|x^{(1)}+x^{(2)}+\cdots+x^{(n)}\right\|_{\ell_{q}^{p}}=n .
$$

As we have remarked earlier, the largest absolute value of $x^{(1)} \pm x^{(2)} \pm \cdots \pm x^{(n)}$ is equal to $n$ for every combination of \pm signs. Moreover, it is clear that for $k \notin\left\{0,2 j, \ldots,\left(2^{n-1}-1\right) j\right\}$, the 
$k$-th term of $x^{(1)} \pm x^{(2)} \pm \cdots \pm x^{(n)}$ is equal to 0 . Hence, we obtain

$$
\begin{aligned}
& \left\|x^{(1)} \pm x^{(2)} \pm \cdots \pm x^{(n)}\right\|_{\ell_{q}^{p}}=\sup _{m \in \mathbb{Z}, N \in \omega}\left|S_{m, N}\right|^{\frac{1}{q}-\frac{1}{p}}\left(\sum_{k \in S_{m, N}}\left|x_{k}^{(1)} \pm x_{k}^{(2)} \pm \cdots \pm x_{k}^{(n)}\right|^{p}\right)^{\frac{1}{p}} \\
& =\sup _{m \in \mathbb{Z} \cap\left[0,\left(2^{n-1}-1\right) j\right], N \in \mathbb{Z} \cap\left[0,\left(2^{n-1}-1\right) j / 2\right]}\left|S_{m, N}\right|^{\frac{1}{q}-\frac{1}{p}}\left(\sum_{k \in S_{m, N}}\left|x_{k}^{(1)} \pm x_{k}^{(2)} \pm \cdots \pm x_{k}^{(n)}\right|^{p}\right)^{\frac{1}{p}}=n .
\end{aligned}
$$

Consequently, we get

$$
\frac{\sum_{ \pm}\left\|x^{(1)} \pm x^{(2)} \pm \cdots \pm x^{(n)}\right\|_{\ell_{q}^{p}}^{2}}{2^{n-1} \sum_{i=1}^{n}\left\|x_{i}\right\|_{\ell_{q}^{p}}}=\frac{2^{n-1} n^{2}}{2^{n-1} n}=n
$$

and

$$
\min \left\|x^{(1)} \pm x^{(2)} \pm \cdots \pm x^{(n)}\right\|_{\ell_{q}^{p}}=n
$$

whence

$$
C_{N J}^{(n)}\left(\ell_{q}^{p}(\mathbb{Z})\right)=C_{J}^{(n)}\left(\ell_{q}^{p}(\mathbb{Z})\right)=n
$$

Case 2: $d>1$. Here we choose $j \in \mathbb{Z}$ to be a nonnegative, even integer such that $j>$ $2^{\left(\frac{n-1}{d}\right)\left(\frac{q}{q-p}\right)}-1$ or, equivalently,

$$
(j+1)^{d\left(\frac{1}{q}-\frac{1}{p}\right)}<2^{-\frac{(n-1)}{p}} .
$$

Then, using the sequences

$$
x^{(i)}=\left(x_{k_{1}}^{(i)}\right)_{k_{1} \in \mathbb{Z}} \in \ell_{q}^{p}(\mathbb{Z}), \quad i=1, \ldots, n,
$$

in the case where $d=1$, we now define $x^{(i)}:=\left(x_{k}^{(i)}\right)_{k \in \mathbb{Z}^{d}} \in \ell_{q}^{p}\left(\mathbb{Z}^{d}\right)$ for $i=1, \ldots, n$, where

$$
x_{k}^{(i)}= \begin{cases}x_{k_{1}}^{(i)}, & k=\left(k_{1}, 0,0, \ldots, 0\right), \\ 0, & \text { otherwise. }\end{cases}
$$

We shall then obtain

$$
C_{N J}^{(n)}\left(\ell_{q}^{p}\left(\mathbb{Z}^{d}\right)\right)=C_{J}^{(n)}\left(\ell_{q}^{p}\left(\mathbb{Z}^{d}\right)\right)=n
$$

as desired.

Corollary 2.2.1. For $1 \leq p<q<\infty$, the space $\ell_{q}^{p}$ is not uniformly non- $\ell_{n}^{1}$.

Corollary 2.2.2. For $1 \leq p<q<\infty$, the space $\ell_{q}^{p}$ is not uniformly $n$-convex.

Acknowledgement. The work is part of the first author's thesis. Both authors are supported by P2MI 2021 Program of Bandung Institute of Technology. 


\section{References}

[1] B. Beauzamy, Introduction to Banach Spaces and Their Geometry, 2nd Ed., North Holland, Amsterdam- New York-Oxford, 1985. https://pascal-francis.inist.fr/vibad/index.php?action=getRecordDetail\&idt= PASCAL82X0319279.

[2] H. Gunawan, D.I. Hakim, A.S. Putri, On geometric properties of Morrey spaces, Ufimsk. Mat. Zh. 13 (2021) 131-136. https://doi.org/10.13108/2021-13-1-131.

[3] H. Gunawan, E. Kikianty, C. Schwanke, Discrete Morrey spaces and their inclusion properties, Math. Nachr. 291 (2018) 1283-1296. https://doi.org/10.1002/mana. 201700054.

[4] H. Gunawan, E. Kikianty, Y. Sawano, and C. Schwanke, Three geometric constants for Morrey spaces, Bull. Korean. Math. Soc. 56 (2019) 1569-1575. https://doi.org/10.4134/BKMS . b190010.

[5] R.C. James, Uniformly non-square banach spaces, Ann. Math. 80 (1964) 542-550. https://doi.org/10.2307/ 1970663.

[6] M. Kato, Y. Takahashi, and K. Hashimoto, On n-th Von Neumann-Jordan constants for Banach spaces, Bull. Kyushu Inst. Tech. 45 (1998), 25-33. https://ci.nii.ac.jp/naid/110000079659.

[7] L. Maligranda, L. Nikolova, L.-E. Persson, T. Zachariades, On n-th James and Khintchine constants of Banach spaces, Math. Inequal. Appl. 1 (2007) 1-22. https://doi.org/10.7153/mia-11-01.

[8] W.A. Wojczynski, Geometry and martingales in Banach spaces, Part II, in: Probability in Banach Spaces IV, J. Kuelbs, ed., Marcel-Dekker, 1978, 267-517. https://doi.org/10.1201/9780429462153. 